\title{
NOVEL SNOW DEPTH RETRIEVAL METHOD USING TIME SERIES SSMI PASSIVE MICROWAVE IMAGERY
}

\author{
Z. Nikraftar ${ }^{\mathrm{a}}$, M. Hasanlou, ${ }^{\mathrm{a},{ }^{*}}$, M. Esmaeilzadeh ${ }^{\mathrm{a}}$ \\ ${ }^{a}$ School of Surveying and Geospatial Engineering, College of Engineering, University of Tehran, Tehran, Iran \\ (xahir.nikraftar, hasanlou, m.esmaeilzade)@ut.ac.ir
}

Commission VIII, WG VIII/6

KEY WORDS: Snow Depth, Support Vector Regression, Nonlinear Modeling, Genetic Algorithm, band combination

\begin{abstract}
:
The Special Sensor Microwave Imager (SSM/I) and the Special Sensor Microwave Imager Sounder (SSM/IS) are satellites that work in passive microwave range. The SSM/I has capability to measure geophysical parameters which these parameters are key to investigate the climate and hydrology condition in the world. In this research the SSMI passive microwave data is used to study the feasibility of monitoring snow depth during snowfall month from 2010 to 2015 using an algorithm in conjunction with ground depth measured at meteorological stations of the National Centre for Environmental Information (NCEI). The previous procedures for snow depth retrieval algorithms uses only one or two passive bands for modelling snow depth. This study enable us to use of a nonlinear multidimensional regression algorithm which incorporates all channels and their related weighting coefficients for each band. Higher value of these coefficients are indicator of the importance of each band in the regression model. All channels and their combination were used in support of the vector algorithm combined with genetic algorithm (GA) for feature selection to estimate snow depth. The results were compared with those algorithms developed by recent researchers and the results clearly shows the superiority of proposed method $\left(\mathrm{R}^{2}=0.82\right.$ and $\left.\mathrm{RMSE}=6.3 \mathrm{~cm}\right)$.
\end{abstract}

\section{INTRODUCTION}

Snow is a key parameter and plays and important role in the water budget and climate change investigation (Winstanley and Wendland, 2007). Furthermore apart from remote sensing there is no precise physical, atmospheric and hydrological model to estimate water equivalent of snow and there results is not reliable. Since the Special Sensor Microwave Imager and sounder SSMI/S is available several studies have demonstrated the capability of space borne passive microwave sensor for monitoring and modeling of the snow properties. The Special Sensor Microwave Imager (SSM/I) and the Special Sensor Microwave Imager Sounder (SSMIS) are satellites that work in passive microwave range. This instruments has been installed onboard Defense Meteorological Satellite Program (DMSP) and are near polar orbiting satellites. Currently F15, F16, F17 and F18 are anther operating("Special Sensor Microwave Imager (SSM/I) Instrument Document. [Online]. Available: https://nsidc.org/data/docs/daac/ssmi_instrument.gd.html Google Search," n.d.). The SSM/I have a seven-channel, four-frequency in two polarization (horizontally and vertically polarized). Actually these sensor measures atmospheric, ocean and terrain microwave brightness temperatures (BT) at 19.35, 22.2, 37.0, and $85.5 \mathrm{GHz}$ in high temporal low spatial resolution. The SSM/I has capability to measure geophysical parameters of ocean wind speed, water vapor in atmosphere, liquid water in cloud, and rain rate (Wentz and Spencer, 1998). It also can be used to model the near surface temperature and humidity. In the past few decades SSM/I passive microwave satellite known as affective way for modeling the snow property's such as snow cover (SC), snow depth (SD), snow water equivalent (SWE). Since in some region precipitation is mostly accrued in snow form, amount of snow fall is indicator of change in hydrological cycle and climate. It is obvious that evaluation and analysis of the snow parameters play an important role in climate research. The most of algorithms for extracting and estimating snow properties retrieval using the passive microwave bands are wrong due to source of the error for example different land cover type and grain size of snow pack. Most of these algorithm assume that land cover (vegetation) does not have any effect on the retrievals algorithm but it is not true. In fact, it can have a significant impact on the accuracy of snow parameters estimation. In this study we mainly focused on estimating of snow depth using different linear and nonlinear algorithms and comparison results with two strategy. Comparison in different monthly and comparison in different snow depth frequencies. In our feature work, we will take into account the involved parameters in snow depth and snow water equivalent retrieval algorithms, such as impact of snow grin

\footnotetext{
* Corresponding author
} 
size and various land cover type on passive microwave responses from snow in various climatic/geographic regions (Zahir and Mahdi, 2015).

\section{STUDY AREA}

The climate of Alaska is a subarctic climate due to its short, cool summers. Average daytime summer temperatures range from approximately 13 to 26 degrees Celsius and average daytime winter temperatures are about -15 to -1 degrees Celsius ("National Centers for Environmental Information (NCEI) - Google Search," n.d.). Alaska in southeast part have a climate of mid-latitude oceanic climate and in the northern parts have a subarctic oceanic climate. In annual scale southeast part is a wettest and warmest part of Alaska with milder temperatures in the winter and high precipitation throughout the year. Average annual precipitation is across from Alaska with total exponentially decreasing from south to north Figure 1. Precipitation in this region is almost in form of snow and for this reason it is too important to be under investigate (Kunkel, 2013).

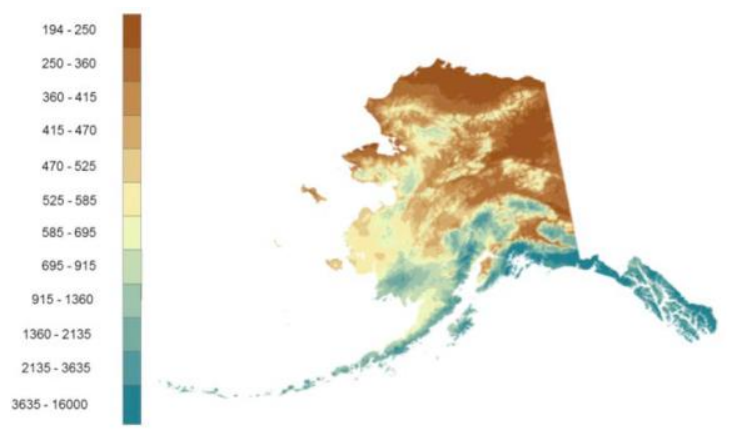

Figure 1. Mean annual precipitation for past decade in Alaska

\section{IMAGES AND DATA SETS}

\subsection{In-situ and ground truth data}

Ground truth measurements of snow depth is available in NOAAS National center for Environmental Information (NCEI) ("Climate," n.d.). Land-based observations are collected from instruments sited at locations in every continent. They measured parameters such as temperature, dew point, relative humidity, precipitation, wind speed, wind direction, visibility, atmospheric pressure, and types of weather occurrences such as hail, fog, and thunder. The NCEI provides a broad level of service associated with landbased observations. These include data collection, quality control, and removal of biases associated with factors such as urbanization and changes in instrumentation through time. Data on sub-hourly, hourly, daily, monthly, annual, and multiyear timescales are available. Ground truth is achieved from snow depth measured by meteorological stations of the National Center for Environmental Information (NCEI) for 37 stations in Alaska (North America) showed in Figure 2. It is important to mention that data sets for all stations is not complete. It means some station have ground measurement snow depth for just one year and these data for all the years was not completed. Number of ground truth is about thirteen thousand samples.

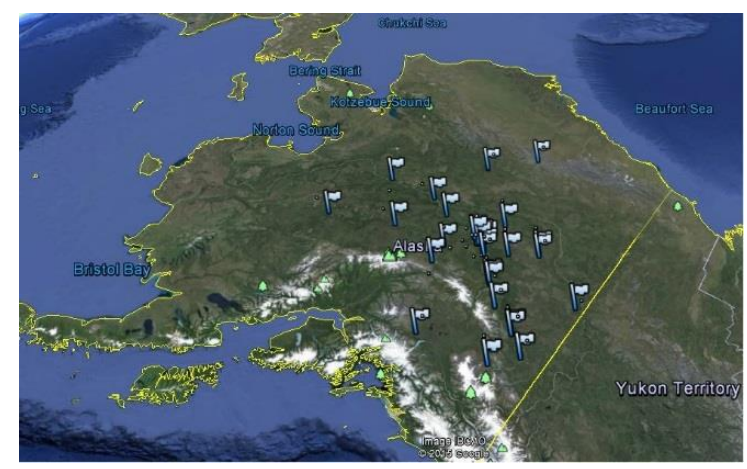

Figure 2. Metrological stations in Alaska 3.2 Passive microwave imagery

As we already discussed, the SSMI passive microwave image data set from 2010 to 2015 in snowfall month (i.e. January, February, November and December) which is simultaneously with ground truth data are used in this study for modeling purposes Figure 3.

Emission in microwave electromagnetic spectrum region for snow cover areas is consist of two types of emissions. 1) Emission from snow itself and 2) Emission from underlying ground Snow crystals essentially scatter part of the cold sky radiation, which reduces the upwelling radiation measured with a radiometer (Schmugge, 1980). There are more crystals in deeper snow packs to scatter microwave emerges. Using these properties of the snow and snow packs and its effects on passive microwave data measuring of the snow parameters is possible. So, snow packs with more than five $\mathrm{cm}$ are able to naturally scattering microwave radiation enough to be detect by passive microwave sensors. For the shallow snow packs (i.e. below five $\mathrm{cm}$ ) the snow will virtually transparent. For this reason we removed ground truth with snow depth less than five cm ("Retrieval of Regional Snow Water Equivalent from Space-Borne Passive Microwave Observations - Google Search," n.d.; Stiles and Ulaby, 1980).

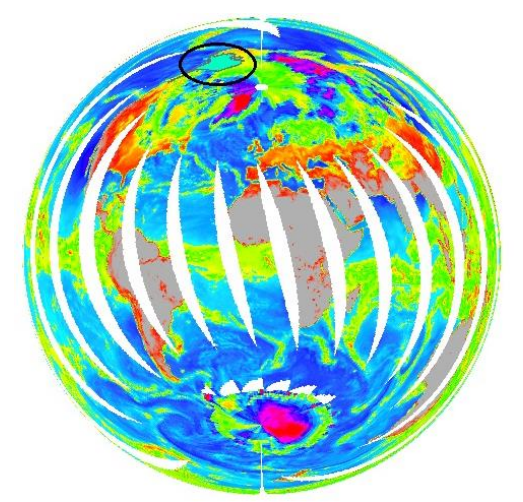

Figure 3. SSMI passive microwave ESA gridded data. We shows Alaska using black elliptic here and in cyan color. 


\subsection{MODIS snow cover products}

Definition of the Snow Cover (SC) areas is the presence of snow over lands in this area. The SC plays an important role in the reflectance of land surface as it is involved in the processes of energy and water exchange between land and atmosphere. The SSMI bands take effect from snow packs and this effect enable us to retrieval snow parameters. If there is snow within the pixels bands takes a different effect from the bare ground and this data cannot be used in snow monitoring. In this regards, we used MODIS (Moderate Resolution Imaging Spectroradiometer) snow cover product for eliminate the snow free pixels from images.

The MODIS/Aqua Snow Cover 8-Day L3 Global 500m Grid (MYD10A2) data set is include maximum snow cover extent in eight-day composite periods and snow occurrence observations in Hierarchical Data Format-Earth Observing System (HDF-EOS) format simultaneously with metadata. MODIS snow cover product for beginning of month March 2013 is shown in Figure 4.

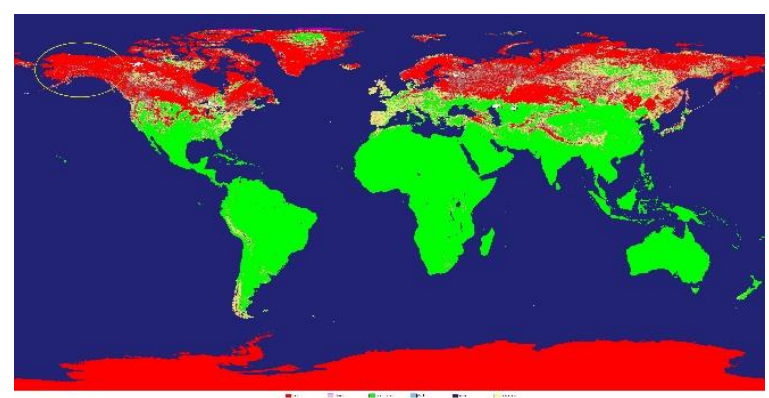

Figure 4. Snow cover areas are shown in red and Alaska is shown here by elliptic acquired by MODIS.

\section{METHODOLOGY}

Snow has a different effect on the brightness temperature of band in passive microwave electromagnetic spectrum. Therefore there are many snow depth retrieval algorithms that used linear combination of the bands to model snow properties (Takala et al., 2011). Most of these algorithm used only one or two bands for modeling snow depth Figure 5. While corporation of the other bands and they combination may have significant or even a little effect on the model improvement. These algorithms are limited because variability in time and space. In different region the retrieval coefficient is static in both space and time and we cannot use one specific model for different regions and different time. As we mentioned before variation of land cover type and climate through time and space is too much in Alaska then it is necessary to implement a novel method that this problem has been resolved in it [5]. In this study nonlinear and kernel based, the SVR (support vector regression) method with incorporating Genetic algorithm (GA) for optimizing purposes (Genetic-SVR) models has been implemented as a high performance method to retrieval snow properties.

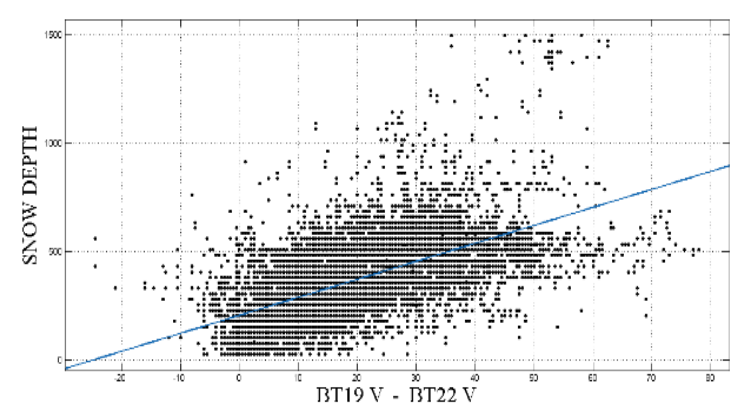

(a) $\mathrm{R}^{2}=0.37$

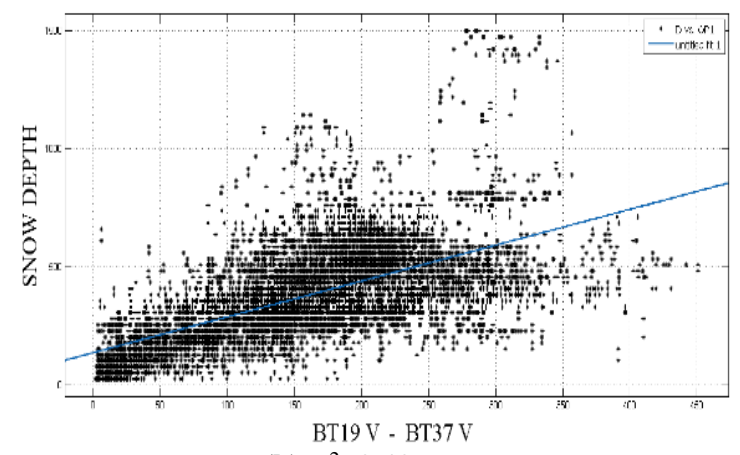

(b) $\mathrm{R}^{2}=0.40$

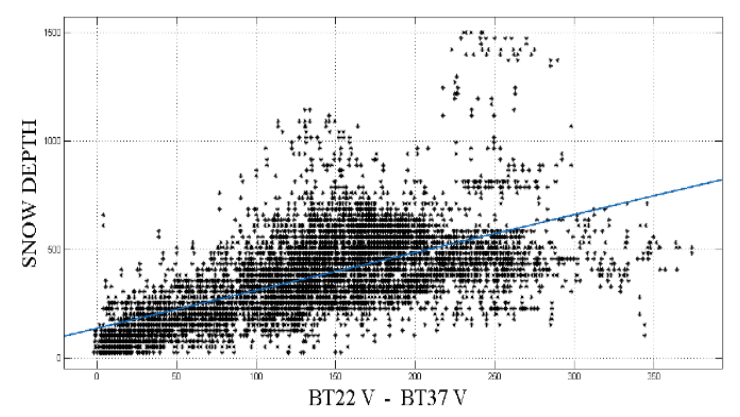

(c) $\mathrm{R}^{2}=0.38$

Figure 5. Brightness temperature difference between (a) B19-B22 and SD, (b) B19-B37 and SD and (c) B22-B37 and SD.

Initial intention of our research is to use all channel (sevenchannel) and their combination to find a suitable algorithm for snow depth modeling. In this regards, by using linear and nonlinear multidimensional regression, we established a novel algorithm (by incorporating all channel and their combination) with related coefficients for each band. Coefficients act like weight. Higher value of these coefficients are indicator of degree of influence of each band in regression model. The second intention is used all channels and their combination in support vector regression (SVR) algorithm combined with genetic algorithm (GA) as a feature selection method (genetically support vector regression) to find a best channel and band for snow depth retrieval.

\subsection{Linear and nonlinear models}

As we mentioned before many snow depth retrieval methods has been presented by others which most of them used 
difference between two bands (between the low and high frequencies) as a retrieval method (Takala et al., 2011). There are many factors in snow internal structure and its surrounding areas which influenced the energy that reached to the sensor. The sensor get different effect from these factors in different bands. Actually by using this property of the passive microwave sensor snow parameters estimation is possible.

We used all channels of the SSMI and they combination in linear and nonlinear (multi-dimensional polynomial of degree 2-5) models to map snow depth. This method will taking into account any possible relation between snow properties and these channels, which result of these models is a coefficients for each band. The coefficients act like weight for each band. Larger (positive or negative) coefficients means the more effectiveness of the bands in retrieval process and vice versa.

\subsection{Support Vector Regression}

The original SVM algorithm was invented by Vladimir N. Vapnik and Alexey Ya. Chervonenkis in 1963. Vapnik suggested a way to create nonlinear classifiers by applying the kernel trick to maximum-margin hyper planes (Boser et al., 1992). The current standard incarnation (soft margin) was proposed by Corinna Cortes and Vapnik in 1993 and published in 1995 (Cortes and Vapnik, 1995). More formally, a support vector machine constructs a hyper plane or set of hyper planes in a high or infinite dimensional space, which can be used for classification, regression, or other tasks. Intuitively, a good separation is achieved by the hyper plane that has the largest distance to the nearest training-data point of any class (so-called functional margin), since in general the larger the margin the lower the generalization error of the classifier. SVR method in the past decade known as one of the reliable and efficient methods. In machine learning, SVMs are supervised learning models which associate with learning algorithm used for classification and regression analysis. The original SVM algorithm was introduced by Vapnik. Scholkopf and Smola proposed a more in-depth overview of SVM regression(Scholkopf and Smola, 2001). SVR for classifying or regression of the multidimensional features takes the data in to higher dimension and using quadratic programing to solve the equation and reduce maximum margin in results. The results of this method in addition to high accuracy has a stability too.

\subsection{Feature selection by Genetic Algorithm}

\subsubsection{Feature Selection}

Feature selection is the process of identifying and removing from a training data set as much irrelevant and redundant features as possible. This, reduces the dimensionality of the data and may enable regression algorithms to operate faster and more effectively. In some cases, correlation coefficient can be improved; in others, the result is a more compact, easily interpreted representation of the target concept (Karagiannopoulos et al., 2007).

\subsubsection{Genetic Algorithm}

Genetic Algorithms (GA) are search algorithms inspired by evolution and natural selection, and they can be used to solve different and diverse types of problems (Mitchell, 1998). The algorithm starts with a group of individuals (chromosomes) called a population. Each chromosome is composed of a sequence of genes that would be bits, characters, or numbers. Reproduction is achieved using crossover (parents are used to produce children) and mutation (alteration of a gene or more). Each chromosome is evaluated using a fitness function, which defines which chromosomes are highly-fitted in the environment. The process is iterated for multiple times for a number of generations until optimal solution or maximum iteration is reached. The reached solution could be a single individual or a group of individuals obtained by repeating the GA process for many runs.

\section{EXPERIMENT AND RESULTS}

Here we exposed two type of strategy's and their analysis which has been used in this study, 1) Snow depth retrieval within snowfall month and 2) Snow depth retrieval in different snow depth frequency.

\subsection{First strategy}

Result of first analyze shown in Table 1 for linear model shows the low performance of this method for snow depth retrieval. Results of this method for months January, February and November are too close, but the result for month December is not close to them. It's maybe because of the influential factors such as snow grain size and land cover type that in our feature works will be investigated. From the Table .1, we can see the improvement of snow depth retrieval R-SQUARE $\left(\mathrm{R}^{2}\right)$ using nonlinear model strategy. Also while the amount of R-SQUARE was increased value of the Root Mean Square Error (RMSE) has been decreased. Multidimensional Polynomial of degree 2-5 has been implemented here and polynomial of degree 3 reached a best results in retrieval process. Also, for G-SVR method amounts of R-SQUARE has been increased significantly. While the amount of R-SQUARE is increased the value of the RMSE is decreased for all four incorporated months.

Table 1. Results of linear, nonlinear and genetically-SVM snow depth retrieval models in different snowfall month

\begin{tabular}{|c|c|c|c|c|c|c|c|c|}
\hline \multirow[t]{2}{*}{ Method } & \multicolumn{2}{|c|}{ January } & \multicolumn{2}{|c|}{ February } & \multicolumn{2}{|c|}{ November } & \multicolumn{2}{|c|}{ December } \\
\hline & R-SQUARE & $\begin{array}{c}\text { RMSE } \\
\mathrm{cm}\end{array}$ & R.SQUARE & $\begin{array}{c}\text { RMSE } \\
\mathrm{cm}\end{array}$ & R-SQUARE & $\underset{\mathrm{cm}}{\text { RMSE }}$ & R.SQUARE & $\begin{array}{c}\text { RMSE } \\
\mathrm{cm}\end{array}$ \\
\hline Linear & 0.27 & 9.8 & 0.30 & 13.6 & 0.32 & 6.7 & 0.17 & 9.6 \\
\hline $\begin{array}{l}\text { Non linear } \\
\text { Poly Degree } \\
2.5\end{array}$ & 0.34 & 9.3 & 0.43 & 12.3 & 0.39 & 6.4 & 0.34 & 8.4 \\
\hline $\begin{array}{c}\text { Genetic } \\
\& \\
\text { SVM }\end{array}$ & 0.67 & 6.4 & 0.71 & 8.1 & 0.65 & 5.9 & 0.69 & 5.7 \\
\hline
\end{tabular}




\subsection{Second Strategy}

In this strategy, we focused on capability of the mentioned algorithm to retrieval of snow depth when we different frequency of the snow depth is used. Purpose of this strategy is to investigate the effects of snow pack depth in brightness temperature $(\mathrm{TB})$ of the passive microwave sensors and performance of retrieval algorithm. Therefore, we used snow depth in four frequency $(30 \mathrm{~cm}, 60 \mathrm{~cm}, 120 \mathrm{~cm}$ and $150 \mathrm{~cm}$ ) and for each frequency randomly, 40 percent of the data sets is used as a training sets and the rested 60 percent is used as a test sets for modeling. It should be notice that in the datasets there is some ground truth more than $150 \mathrm{~cm}$. But number of these ground truth observation is too low and it will make perturbation in the retrieval processes. For this reason we removed these observations from the datasets to prevention of egregious error. Since with variation in snow depth size of grin size and snow pack liquid water content, will increased so obviously capability of the algorithm will be different in different frequency. The results for linear model as Table 2 shows have maximum R-SQUARE about 55 percent for $60 \mathrm{~cm}$ frequency. When frequency was increased amount of R-SQUARE was decreased. These variations in value of the R-SQUARE is because of the variations in snow grain size and liquid water content of snow. Amount of R-SQUARE for nonlinear and G-SVR increased significantly. This result shows that the relation between snow depth and passive microwave data is more tend to nonlinear relation rather than linear relation. Convergence of the Genetically-SVM model has been shown here in Figure 6.

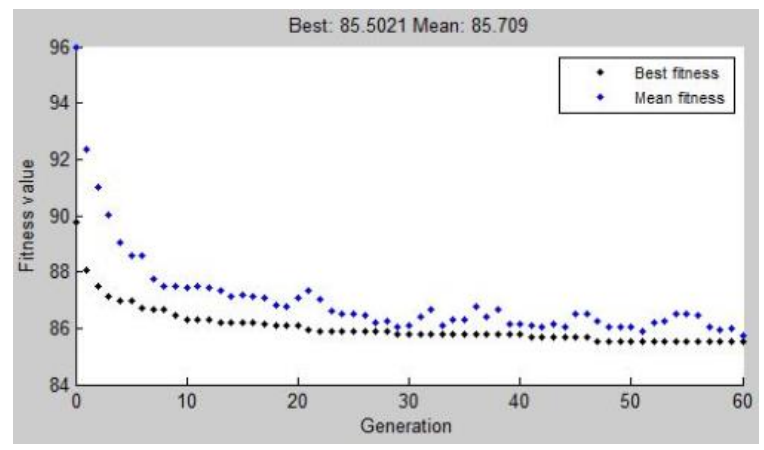

Figure 6. Convergence of Genetically-SVM

Table 2. Snow depth retrieval result for different snow frequency using linear, nonlinear, genetically SVR.

\begin{tabular}{ccccccccc} 
Method & $\mathrm{SD}<30 \mathrm{CM}$ & $\mathrm{SD}<60 \mathrm{CM}$ & $\mathrm{SD}<120 \mathrm{CM}$ & $\mathrm{SD}<150 \mathrm{CM}$ \\
\hline & R-SQUARE & $\begin{array}{c}\text { RMSE } \\
\mathrm{cm}\end{array}$ & R-SQUARE & $\begin{array}{c}\text { RMSE } \\
\mathrm{cm}\end{array}$ & R-SQUARE & $\begin{array}{c}\text { RMSE } \\
\mathrm{cm}\end{array}$ & R-SQUARE & $\begin{array}{c}\text { RMSE } \\
\mathrm{cm}\end{array}$ \\
\hline Linear & 0.45 & 5.6 & 0.55 & 10 & 0.53 & 12 & 0.51 & 13 \\
\hline $\begin{array}{c}\text { Non linear } \\
\text { Poly Degre 2 }\end{array}$ & 0.55 & 5 & 0.66 & 8.7 & 0.63 & 11 & 0.61 & 12 \\
\hline $\begin{array}{c}\text { Genetic } \\
\text { \& } \\
\text { SVM }\end{array}$ & 0.70 & 4.2 & 0.82 & 6.3 & 0.81 & 7.9 & 0.80 & 8.7 \\
\hline
\end{tabular}

\section{CONCLUSION}

In this study we propose two new passive microwave SD retrieval algorithm based on nonlinearity of the relations between passive microwave channels and their combinations, with ground truth snow depth measurements and implement these in Alaska-North America in the period of 2010 until 2015 in snowfall month. Although there is a significant error because variations of snow grain size but the assumption of constant grain size is considered here. Investigation of its effect on snow depth retrieval algorithm will be our future study. We used three snow depth retrieval algorithm and two strategies for evaluation capability of the SSMI passive microwave data in snow monitoring. The retrieval algorithms are including linear, nonlinear and Genetically-SVR and the strategies are snowfall analysis in different month and snowfall analysis in different frequency of snow depth. Results indicate that the retrieval of snow depth from space borne passive microwave observations can be improved by using nonlinear (kernel based) models while 40 percent of the data is used as training set and the rest of the data (60 percent) as a test and validation. Also analyzing of the band shows, using only spectral differences is not effective method for snow depth monitoring while using spectral band and their combination simultaneously is much more effective and reliable. Results shows that nonlinear multidimensional polynomial have a more better performance compare to linear model and G-SVR retrieval algorithm have higher performance rather than other method. Performance of the retrieval algorithm in first strategy is lesser than second strategy. It is because of variation in snow depth and consequently snow grain size. Variations of snow pack depth have direct and significant effect on emitted energy to the sensor and this issue was impressed retrieval algorithms.

\section{References}

Boser, B.E., Guyon, I.M., Vapnik, V.N., 1992. A training algorithm for optimal margin classifiers, in: Proceedings of the Fifth Annual Workshop on Computational Learning Theory. ACM, pp. 144152.

Climate [WWW Document], n.d. URL http://www.muni.org/FastFacts/Pages/Climate.as px (accessed 1.7.16).

Cortes, C., Vapnik, V., 1995. Support-vector networks. Mach. Learn. 20, 273-297.

Karagiannopoulos, M., Anyfantis, D., Kotsiantis, S.B., Pintelas, P.E., 2007. Feature selection for regression problems. Proc. 8th Hell. Eur. Res. Comput. Math. Its Appl. Athens Greece 2022.

Kunkel, K.E., 2013. Regional climate trends and scenarios for the US national climate assessment. US Department of Commerce, National Oceanic and Atmospheric Administration, National Environmental Satellite, Data, and Information Service.

Mitchell, M., 1998. An introduction to genetic algorithms. MIT press.

National Centers for Environmental Information (NCEI) Google Search [WWW Document], n.d. URL https://www.google.com/?gws_rd=ssl\#q=Nationa 
1+Centers+for+Environmental+Information+(NC EI) (accessed 1.7.16).

Retrieval of Regional Snow Water Equivalent from SpaceBorne Passive Microwave Observations - Google Search [WWW Document], n.d. URL https://www.google.com/search?q=Retrieval+of+ Regional+Snow+Water+Equivalent+from + Space

Borne+Passive+Microwave+Observations\&biw $=$ 1366\&bih $=596 \&$ ei $=487 \mathrm{tVvbeAqzN6QTDiryICg}$ $\&$ start $=0 \& s a=\mathrm{N}$ (accessed 3.19.16).

Schmugge, T.J., 1980. Microwave approaches in hydrology.

Scholkopf, B., Smola, A.J., 2001. Learning with kernels: support vector machines, regularization, optimization, and beyond. MIT press.

Special Sensor Microwave Imager (SSM/I) Instrument Document. [Online]. Available: https://nsidc.org/data/docs/daac/ssmi_instrument. gd.html - Google Search [WWW Document], n.d. URL

https://www.google.com/search?q=Special+Sens or+Microwave+Imager+(SSM/I)+Instrument+Do cument.+\%5BOnline\%5D.+Available:+https://nsi dc.org/data/docs/daac/ssmi_instrument.gd.html\& sa $=$ X\&ved=0ahUKEwj30vq64srLAhUhMZoKH d0KD6sQgwMIGw\&biw=1366\&bih=596 (accessed 3.18.16).

Stiles, W.H., Ulaby, F.T., 1980. The active and passive microwave response to snow parameters: 1 . Wetness. J. Geophys. Res. Oceans 85, 10371044.

Takala, M., Luojus, K., Pulliainen, J., Derksen, C., Lemmetyinen, J., Kärnä, J.-P., Koskinen, J., Bojkov, B., 2011. Estimating northern hemisphere snow water equivalent for climate research through assimilation of space-borne radiometer data and ground-based measurements. Remote Sens. Environ. 115, 3517-3529.

Wentz, F.J., Spencer, R.W., 1998. SSM/I rain retrievals within a unified all-weather ocean algorithm. J. Atmospheric Sci. 55, 1613-1627.

Winstanley, D., Wendland, W.M., 2007. Climate change and associated changes to the water budget. Clim. Change Var. Primer Teach. 1.

Zahir, N., Mahdi, H., 2015. SNOW DEPTH ESTIMATION USING TIME SERIES PASSIVE

MICROWAVE IMAGERY VIA GENETICALLY SUPPORT VECTOR REGRESSION (CASE STUDY URMIA LAKE BASIN). Int. Arch. Photogramm. Remote Sens. Spat. Inf. Sci. 40, 555. 